\title{
JUGO DE PAPAYA INMADURO PARA TRATAR LA ULCERA GÁSTRICA EN ESTUDIANTES DE LA UNIVERSIDAD PRIVADA DE HUANCAYO
}

Autores:

Junchaya Yllescas, V.; Andamayo Flores D.

\section{RESUMEN}

En el Perú la ulcera gástrica es una enfermedad frecuente en el sexo masculino y en edades tempranas de la vida, y no hay diferencias entre las poblaciones de la costa, la sierra y la selva del Perú, siendo el objetivo general de esta investigación evaluar cómo el Jugo de papaya inmaduro puede tratar la Ulcera Gástrica en estudiantes de la UPH y como objetivos específicos determinar el porcentaje de estudiantes que padece de úlcera gástrica, cuáles son los principales síntomas y que metabolitos secundarios principales contiene el jugo de papaya inmaduro, realizado en el consultorio médico y laboratorio de investigación de la UPH en 301 estudiantes ingresantes de las carreras de Ciencias Farmacéuticas y Bioquímica, Enfermería y obstetricia, mediante un estudio descriptivo de recogida de casos clínicos encontrándose como resultado que el $25 \%$ presentan síntomas de úlcera gástrica, siendo los más frecuentes las regurgitaciones ácidas hacia la boca (16\%), dolor localizado en el epigastro $(15 \%)$ y pirosis $(13 \%$ y los metabolitos secundarios principales fueron los terpenoides, alcaloides, flavonoides, carbohidratos, saponinas y sustancias esteroidales en magnitud de +++ .

\section{Palabras claves: Carica papaya, ulcera gástrica}

\section{SUMMARY}

In Peru, gastric ulcer is a common disease in males and at early ages of life, and there is no difference between the populations of the coast, highlands and jungle of Peru, with the overall objective of this research to assess how the unripe papaya juice can treat gastric Ulcer in UPH students and specific objectives determine the percentage of students who suffer from gastric ulcer, which are the main symptoms and major secondary metabolites containing the unripe papaya juice, held at the medical office and research laboratory at $301 \mathrm{UPH}$ racing incoming students of Pharmaceutical Sciences and Biochemistry, Nursing and midwifery, including a description of clinical cases collected as a result finding that $25 \%$ of gastric ulcer symptoms, with the Frequently the acid regurgitation into the mouth (16\%), located on the epigastrium pain $(15 \%)$ and heartburn $(13 \%$ and the main secondary metabolites were terpenoids, alkaloids, flavonoids, carbohydrates, saponins and steroidal substances in magnitude +++ . Keywords: Carica papaya, gastric ulcer.

\section{INTRODUCCIÓN}

La úlcera gástrica o de estómago es una erosión en la mucosa gástrica que, con frecuencia, tiene unos tres centímetros de diámetro, aunque no se conoce las causas que la producen, se ha comprobado que está asociada con la irritación de la mucosa gástrica.

Una úlcera gástrica puede producir una hemorragia de estómago. Aunque esto no resulta común, puede resultar peligrosa, ya que puede 
causar un shock, si es repentina, o una anemia si se prolonga durante mucho tiempo. Si la úlcera no se trata, puede ser causa de una importante pérdida de peso y desnutrición, que derivan de la pérdida de apetito. Todo ello predispone al organismo a contraer infecciones. Por otro lado durante la educación universitaria se produce grandes cambios de metodología de trabajo académico en los estudiantes ocasionando en ellos dificultades de saber organizar el tiempo produciendo como consecuencia abandono en consumir los alimentos fundamentales a las horas establecidas y lo que inicialmente puede ser una úlcera sino es tratada a tiempo, puede derivar en un cáncer de estómago reportado incidencias que van desde un $2,4 \%$ hasta un $8 \%$ según las diferentes series en adultos jóvenes menores de 40 años.

El tratamiento que se utiliza para combatir la ulcera gástrica es casi siempre intervenciones quirúrgicas que permita cerrar lesiones, así como algunos fármacos que controlan el ácido cada vez mejor, con antibióticos que trabajando juntos a los bloqueadores de la bomba de protones (BBP) o al bismuto cicatrizan las úlceras y erradican al $\mathrm{H}$. pylori en más del $90 \%$ de los casos; con tecnología endoscópica de diagnóstico cada vez más precisa; e inclusive con técnicas quirúrgicas mejoradas, la enfermedad ulcerosa péptica debería de ser un problema cada vez menor para el gastroenterólogo.

Sin embargo la papaya es una de las frutas más apreciadas en las regiones tropicales, no solo por su delicioso sabor, sino también por sus cualidades medicinales ya que favorece la digestión debido a que contiene papaina, y de acuerdo al investigador Ezike, A. (2010) ${ }^{3}$ ha demostrado que el jugo de papaya verde es un potencial anti ulceroso porque además ayuda a inhibir la motilidad intestinal. En el marco metodológico para la recopilación de información, se utilizó el estudio de tipo descriptivo y tecnológico porque permitió registrar situaciones

\begin{tabular}{lcc}
\hline CARRERAS & \multicolumn{2}{c}{ SÍNTOMAS } \\
\hline & \multicolumn{1}{c}{ SI } & NO \\
\cline { 2 - 3 } $\begin{array}{l}\text { Ciencias Farmacéuticas } \\
\text { Bioquímica } \\
\text { Enfermeria }\end{array}$ & & \\
Obstetricia & $25 \%$ & $75 \%$ \\
\hline
\end{tabular}

n: 301

Fuente: Evaluación médica realizada a los estudiantes ingresantes de la $\mathrm{UPH}$

En la tabla 3 se aprecia que el porcentaje de estudiantes que presentan síntomas de úlcera gástrica son el $25 \%$ (75), a diferencia que un $75 \%$ (226) de ellos no lo presentan. y eventos de los estudiantes ingresantes en el año 2012, así como medir y evaluar diversos aspectos, dimensiones o componentes de la ulcera gástrica, información que una vez recogida y procesada fue estructurada diversos capítulos. ${ }^{8}$

\section{OBJETIVO}

Evaluar cómo el Jugo de papaya inmaduro puede tratar la Ulcera Gástrica en estudiantes de la Universidad Privada de Huancayo.

\section{METODOLOGÍA DE LA INVESTIGACIÓN}

La investigación corresponde al tipo descriptivo recogida de casos clínicos de los estudiantes ingresantes del año 2012. Según el tiempo en que se efectuó se considera una investigación sincrónica porque se estudió en el ingreso de cachimbos 2012-I y 2012-II. Asimismo de acuerdo a su proyección en el tiempo esta investigación fue considerada como prospectiva, porque se inicia con la detección de la úlcera gástrica.

De acuerdo a la naturaleza de la información que se recoge para responder al problema de investigación el presente trabajo fue de tipo cuantitativo y cualitativo porque permite registrar información de metabolitos secundarios presentes. El nivel de investigación es descriptivo para especificar o registrar información sobre las manifestaciones clínicas.

El lugar de la investigación se realizó en el consultorio médico y laboratorio de investigación de la Universidad Privada de Huancayo Franklin Roosevelt. Corresponde a un diseño descriptivo, transversal cuantitativo.

\section{Población y muestra}

La población correspondió en aproximadamente 478 estudiantes ingresantes en Ciencias de la Salud y durante el año 2012, cuya muestra fue probabilística ajustada de 304 estudiantes, los cuales fueron seleccionados por padecer úlcera gástrica y según la evaluación médica y considerados en tres grupos según las carreras de Enfermería, Ciencias Farmacéuticas y Bioquímica y Obstetricia empleándose para estratificar el factor multiplicador de 0.63 y como se detalla:

\section{RESULTADOS}

Una vez procesada y analizada la información se obtuvieron los siguientes resultados:

Tabla 3: Porcentaje de estudiantes que presentas síntomas de úlcera gástrica 
Tabla 4: Cantidad de síntomas Clínicos principales sobre úlcera gástrica que presentan los estudiantes de la UPH.

\begin{tabular}{|c|c|c|c|c|}
\hline \multirow{3}{*}{ PRINCIPALES SÍNTOMAS } & \multicolumn{4}{|c|}{ I CICLO } \\
\hline & \multicolumn{2}{|r|}{ SI } & \multicolumn{2}{|c|}{ No } \\
\hline & $\mathrm{N}^{0}$ & $\%$ & $\mathrm{~N}^{0}$ & $\%$ \\
\hline Regurgitaciones ácidas hacia la boca & 40 & $15 \%$ & 35 & $10 \%$ \\
\hline Dolor epigástrico & 43 & $16 \%$ & 32 & $10 \%$ \\
\hline Sensación de estar satisfecho & 29 & $11 \%$ & 46 & $14 \%$ \\
\hline Pirosis sensación de quemazón & 35 & $13 \%$ & 40 & $12 \%$ \\
\hline Nauseas en las primeras horas mañana & 31 & $12 \%$ & 44 & $13 \%$ \\
\hline Cansancio y malestar & 27 & $10 \%$ & 48 & $14 \%$ \\
\hline Pérdida de Apetito & 32 & $12 \%$ & 43 & $13 \%$ \\
\hline Pérdida de peso & 28 & $11 \%$ & 47 & $14 \%$ \\
\hline TOTAL & 265 & $100 \%$ & 335 & $100 \%$ \\
\hline
\end{tabular}

En la tabla 4 se puede apreciar que las manifestaciones clínicas sobre úlceras gástricas que presentaron los estudiantes son regurgitaciones ácidas hacia la boca $(16 \%)$ seguido de dolor epigástrico (15\%) y pirosis o sensación de quemazón en el $(13 \%)$.

Tabla 5: Presencia de metabolitos secundarios que contiene el jugo de papaya inmaduro

En la tabla 4 se puede apreciar que las manifestaciones clínicas sobre úlceras gástricas que presentaron los estudiantes son regurgitaciones ácidas hacia la boca (16\%) seguido de dolor epigástrico (15\%) y pirosis o sensación de quemazón en el (13\%).

Tabla 5: Presencia de metabolitos secundarios que contiene el jugo de papaya inmaduro

\begin{tabular}{lcc}
\multicolumn{1}{c}{$\begin{array}{c}\text { Prueba de } \\
\text { caracterización }\end{array}$} & Metabolito secundario & Calificación \\
\hline $\begin{array}{l}\text { Reacción de Lieberman- } \\
\text { Burchard }\end{array}$ & Terpenoides, & +++ \\
$\begin{array}{l}\text { Reacción de Shinoda } \\
\text { Prueba de la espuma }\end{array}$ & Flavonoides & +++ \\
$\begin{array}{l}\text { Reacción del tricloruro } \\
\text { férrico }\end{array}$ & Saponinas & +++ \\
Reacción de Dragendorff & Alcaloides & +++ \\
$\begin{array}{l}\text { n: } 301 \\
\text { Fuente: Evaluación médica realizada a los estudiantes ingresantes de la UPH }\end{array}$
\end{tabular}

En la tabla 5 se puede apreciar que frente al estudio fitoquímico para determinar la presencia de metabolitos secundarios, se encuentra que los terpenoides, flavonoides, saponinas, compuestos, flavonoides, saponinas, compuestos fenólicos y alcaloides están en una magnitud de +++ .

\section{DISCUSIÓN}

Los resultados de la investigación demostraron que entre los metabolitos secundarios destacados en magnitud +++ en el fruto inmaduro o verde de papaya se encontraron los terpenoides, alcaloides, flavonoides, carbohidratos, saponinas y sustancias esteroidales, los mismos que de acuerdo a un estudio último llevado a cabo en la Universidad de Nigeria, ha demostrado el potencial antiulceroso de los extractos acuoso con una reducción de las lesiones realmente significativas comparado a un grupo control $(\mathrm{P}<05)$. (tabla 5$)^{1}$.
En cambio otro estudio realizado por Perez, S.; Zavaleta, M.; Gonzales, M.; Ortega, N. realizado en México encontraron diecinueve compuestos químicos en las semillas siendo los principales el ácido oleico $(45,97 \%)$, palmítico $(24,1 \%)$ y los ácidos esteárico de $(8,52 \%)$.

Se debe a futuro muy corto proseguir los estudios de esta investigación para favorecer reducir la sintomatología presentada en el $25 \%$ (75) de los estudiantes universitarios tabla 3, ya que la presencia de alcaloides por ser compuestos nitrogenados complejos tiene la propiedad de formar sales con los ácidos y que actúan sobre el sistema nervioso, primero excitándolo y luego paralizándolo, originando la desinflamación de mucosas. ${ }^{4,5,6}$ En el caso de los terpenoides por contener aceites esenciales son excelentes antibacterianas y antifúngicas, en el caso de los flavonoides son poderosos antinflamatorias y antioxidante como los compuestos fenólicos. Además Okewimi; A. demostró que las semillas de la Carica papaya protege la mucosa gástrica reduciendo significativamente el volumen de jugo gástrico y la acidez gástrica $(p<0.05)$ por contener terpenoides y que el extracto de la semilla de papaya C. puede poseer gastro efectos protectores contra la úlcera gástrica de etanol inducida en ratas macho. ${ }^{2,3,4}$

Además es preocupante encontrar de acuerdo a tabla 4 que los síntomas más importantes que presentaron los estudiantes fueron regurgitaciones ácidas hacia la boca $(16 \%)$ seguido de dolor localizado en el epigastro (15\%) y pirosis, cuando Vidal, J. refiere que si las regurgitaciones contienen bilis incrementa las ulceraciones, de allí que se justifica que el dolor epigástrico es otro de los síntomas que más padecen los estudiantes universitarios. ${ }^{8,9,10}$ Enfermedad que de acuerdo a Ormachea señala que en algunos casos las úlceras son asintomáticas o dan pocas molestias y se diagnostican tras la aparición de una complicación como Hemorragia digestiva con vómito de sangre roja, perforaciones y Estenosis pilórica, y que puede resultar fatal para la población de allí que debe ser fundamental el seguimiento médico.

\section{CONCLUSIONES}

1. La cantidad de síntomas clínicos que presentaron los estudiantes de la UPH fueron que un $25 \%$ de ellos presentaron síntomas de ulcera gástrica y en las carreras de Enfermería, Obstetricia y enfermería.

2. Los síntomas encontrados principalmente fueron, regurgitaciones ácidas hacia la boca $(16 \%)$ seguido de dolor localizado en el epigastro $(15 \%)$ y pirosis $(13 \%)$.

3. Los metabolitos secundarios principales identificados en el jugo de papaya inmaduro, fueron en magnitud +++ los 
terpenoides, alcaloides, flavonoides, carbohidratos, saponinas y sustancias esteroidales,

\section{REFERENCIAS BIBLIOGRÁFICAS}

1. Pérez, S.; Zavaleta, M.; Gonzales, M.; Ortega, N. Bioactividad de Carica papaya (Caricaceae) contra Spodoptera frugiperda (Lepidoptera: Noctuidae). Departamento de Sistemas Biológicos, Universidad Autónoma Metropolitana-Xochimilco, Calzada del Hueso 1100, Col. Villa Quietud, México DF 04960, México.

2. Ortega, A.; Jímenez, M.; Acosta, V. Et al. Efectos de semillas de la papaya se extracción en las características del esperma de perros. Departamento de Veterinaria y medicina preventiva, Facultad de Medicina Veterinaria y Ciencia Animal, Universidad Autónoma de Yucatán, Mérida Yucatán, Yucatán, México. 2011.

3. Ezike, A.; Akah,P.;Okoli,E.; Ezeuchenne,N. Fruta inmadura de Carica papaya (papaya) puede ser beneficioso en la úlcera. Departamento de Farmacología y Toxicología de la Facultad de ciencias farmacéuticas, Universidad de Nigeria, Nsukka, estado de Enugu, Nigeria. 2008.

4. Adekunle, A. La actividad Anti-ulcerogenic del extracto acuoso de la fruta de la Papaya Carica en aspirina - inducida por úlcera en ratas Agustín Universidad de Isaac A. y Universidad de Benin ciudad de Benin Nigeria.2008.

5. Gastro-protective activity of aqueous Carica papaya seed extract on ethanol induced gastric ulcer in male rats

6. Helicobacter Pilory en el Perú. Órgano O ficial de la Sociedad de Gastroenterología del Perú. Fondo Editorial de la Universidad Peruana Cayetano Heredia. Perú.2003.

7. Ormachea, E. Úlcera Gástrica. Disponible en la URL.

http://www.mapfre.com/salud/es/cinformat ivo/ulcera-gastrica.shtml(Fecha de acceso 26 de Enero 2013.)

8. Brizuela RA, Fabregas $C$, Angulo $O$, Pérez M, García E, Díaz ME. Helicobacter pylori y enfermedad ulcerosa. Rev Cubana Med Milit 1999.

9. Brunton L. Fármacos para el control de la acidez gástrica y el tratamiento de ulceras pépticas. México: Mc Graw - Hill Interamericana; 1998.

10. Crawford JM. Aparato gastrointestinal. En:
Cotran RS, Kumar V, Collins T, directores. Patología estructural y funcional. 6. a ed. Madrid: Mc Graw - Hill - Interamericanal. 1999.

11. Carson, R. Factores de Riesgo para enfermedades de úlceras gástricas. Daus Mahnke. 2010.

12. Doll, citado por Wayar, T. Úlcera gástrica. Actualización Terapéutica. Editorial Interamericana - McGraw - Hill.Año. 2003.

13. Duarte, J. Anatomía Patológica del Aparato Digestivo. (Fecha de acceso 2 de Noviembre del 2012). Disponible en la web:http://escuela.med.puc.cl/paginas/pu blicaciones/anatomiapatologica/04digesti vo/4estomago_1.html\#ulcera

14. Rodríguez, U. Ülceras gastrointestinales. Campylobacter pilórico, gastritis crónica, duodenitis crónica, úlcera gástrica y úlcera duodenal. Arq Gastroenterol Sao Paulo 1987; 24(1): 10-15.

15. Chan Frances KL, Leung WK. Peptic-ulcer disease.Lancet 2002; 360:933-941.

16. McKay AJ, McArdel CS. Ciimetidine and perforated peptic ulcer. Br J Surg. 1982; 69: 319-320

17. McCownell DB; Baba GC, Deveney CW. Changes in surgical treatment of peptic ulcer disease within a Veterans Hospital in the 1970s and the 1980s. Arch Surg. 1989; 124: 1164-1167.

18. Recavarren-Arce S, León-Barúa R, Cok J, et al. Low prevalence of gastric metaplasia in the duodenal mucosa in Peru. J Clin Gastroenterol 1992; 15: 296-301.

19. Davenport HW. Gastric mucosal hemorrhage in dogs. Effects of acid, aspirin and alcohol. Gastroenterol 1999; 56:439-449.

20. Elliot SN, McKnight W, Cirino G, Wallace $\mathrm{JL}$. A nitricoxide nonsteroidal antiinflammatory drug accelerate gastric ulcer healing in rats. Gastroenterol 1995; 109: 524-30)

21. Behrman Stephen Ramírez-Ramos A, Chinga Alayo E, Mendoza Requejo D, et al. Variación de la prevalencia de $\mathrm{H}$. pylori en el Perú, período 1981-2002. Nivel socioeconómico medio y alto. Rev. de Gast. del Perú. 2003; 23:92-98

Fecha de recepción: 02/04/2013

Fecha de aprobación: 08/04/2013

Correspondencia: Amparo Junchaya Yllescas

Email: amparojunchaya@hotmail.com 